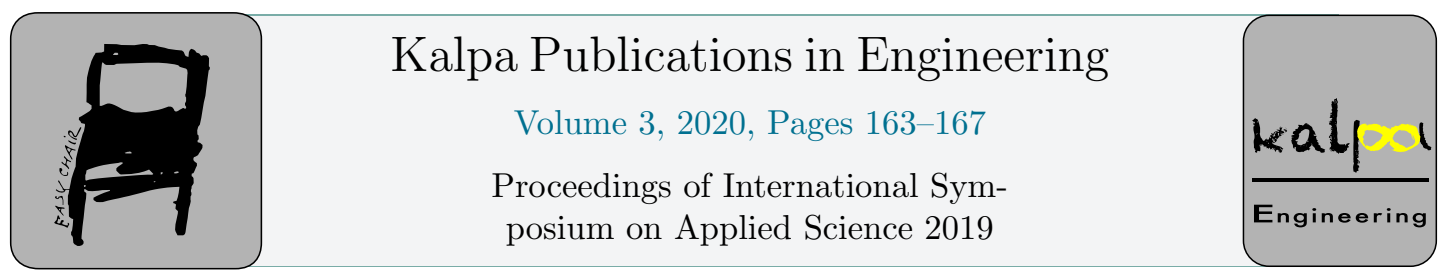

\title{
Optimal Vertically Ascending Flight of an Insect-Like Flapping-Wing Micro Air Vehicle
}

\author{
Anh Tuan Nguyen ${ }^{1}$, Hoang Quan Dinh ${ }^{1}$, Van Thang Nguyen ${ }^{1}$, Vu Dan \\ Thanh Le ${ }^{1}$, Thanh Dong Pham ${ }^{1}$, Dinh Quang Nguyen ${ }^{2}$ \\ ${ }^{1}$ Le Quy Don Technical University, Ha Noi, Vietnam. \\ ${ }^{2}$ Institute of Technology, General Department of Defense Industry, Ha Noi, Vietnam. \\ anhtuannguyen2410@gmail.com
}

\begin{abstract}
This paper explores the optimal flight condition of an insect-like flapping-wing micro air vehicle (FWMAV) while ascending vertically at constant speeds. The FWMAV is assumed to have the same mass properties and wing geometry as those of the hawkmoth Manduca sexta. The optimization is conducted through the combination of an artificial neural network and the genetic algorithm. The training data for the artificial neural network are provided by the unsteady vortex-lattice method written in the programming language FORTRAN using parallel computation techniques. The results show that the FWMAV has to alter its wing kinematics and flapping frequency to sustain vertically ascending flight. Moreover, while ascending, the FWMAV requires more energy than that in hover. The findings from this work are useful for the design of control strategies used for insect-like FWMAVs
\end{abstract}

\section{Introduction}

Insect-like flapping-wing micro air vehicles (FWMAVs) belong to a new type of small-scale aircraft that can mimic the flight mechanisms of insects in wild. One of the most prominent advantages of insect-like FWMAVs over other types of flying vehicles is the ability to operate in confined space, as well as possessing a high level of maneuverability. There have been many studies on the characteristics of insect-like FWMAV flight performance (Wei, et al., 2010) (Liu, Ravi, Kolomenskiy, \& Tanaka, 2016) (Nguyen, Han, \& Han, 2017). Most of these studies have focused only on the hovering and forward flight states, while ascending flight has not been investigated. In nature, ascending flight can be observed while insects are taking off. For FWMAVs, this flight state is important and should be considered for the design process.

In this paper, optimal vertically ascending flight is studied for the first time using the genetic algorithm. An artificial neural network (ANN) is employed to rapidly provide data for the genetic algorithm. The training data for the ANN is obtained from the extended unsteady vortex-lattice 
method (UVLM). The paper shows the relationship between the wing kinematics and the ascending flight speed of the FWMAV.

\section{FWMAV Model and Methodology}

\subsection{FWMAV Model}

The insect-like FWMAV is assumed to have the same mass and geometric properties of the hawkmoth Manduca sexta (Figure 1a). Two wings are connected to the body by two spherical joints. The FWMAV has mass of $1.58 \mathrm{~g}$ and wing length of $4.85 \mathrm{~cm}$.

The position of a wing is defined by the set of three Euler angles, which are $\phi$ (the sweep angle), $\theta$ (the elevation angle) and $\alpha$ (the rotation angle). The definitions of these angles are shown in Figure 1b. $\beta$ is the stroke plane angle, and it is assumed that the angle between the stroke plane and the body axis is held constant at $120^{\circ}$.

The variations of the Euler angles are as follows:

$$
\begin{aligned}
& \phi=\phi_{a} \sin \left(2 \pi f t+\frac{3 \pi}{2}\right)+\phi_{0} \\
& \theta=\theta_{0} \\
& \alpha=\alpha_{0}-\alpha_{a} \sin (2 \pi f t)
\end{aligned}
$$

where subscripts 0 and $a$ denote the mean value and the amplitude, respectively. $f$ is the flapping frequency.

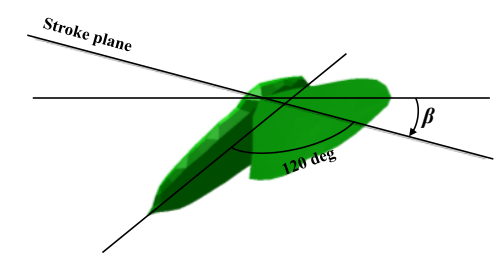

a)

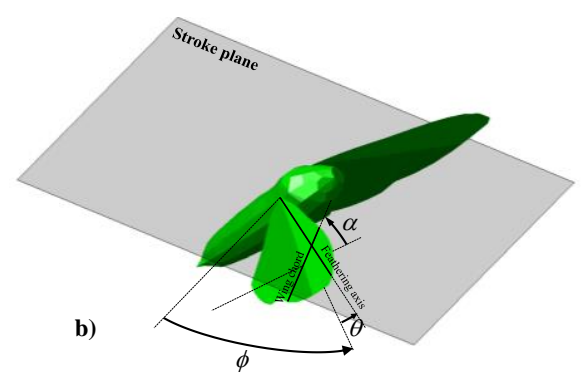

Figure 1: FWMAV and angle definitions

\subsection{Methodology}

In this study, the optimal equilibrium flight state corresponding to each vertical speed is obtained by the use of the genetic algorithm (Mitchell, 1998). It is noted that the objective of the optimization is to minimize the required mechanical power while the equilibrium conditions are satisfied, which means the mean lift must be equal to the weight of the FWMAV, and the mean drag and pitch moment about the mass center are zero. The constrained optimization problem could be tranformed to a non-constrained problem by the use of the penalty function: 


$$
F=\frac{\bar{P}}{P_{0}}+r\left(\left|1-\frac{\bar{L}}{W}\right|+\left|\frac{\bar{D}}{W}\right|+\left|\frac{\bar{M}}{W \bar{c}}\right|\right)
$$

Here, $F$ is the fitness function, $\bar{L}, \bar{D}, \bar{M}$ and $\bar{P}$ are respectively the mean lift, drag, pitch moment and power. $P_{0}$ is the reference power, which is equal to the mean lift required for an actual hawkmoth while hovering. $P_{0}$ is $84.2 \mathrm{~mW}$ (Nguyen, et al., 2019). $W$ is the weight of the FWMAV. $r$ is a constant that is set to 2.0 in this study.

There are seven parameters that are considered in the optimization problem, including $\phi_{a}, \phi_{0}, \theta_{a}, \theta_{0}, \alpha_{a}, \alpha_{0}$ and $\beta$.

Initially, a random population is generated, and the fitness value of each individual is determined. Next, through the mutation and crossover processes, this population keeps evolving from generation to generation. The evolution stops when the optimal solution is obtained.

It should be noted that the genetic algorithm requires a huge number of fitness evaluations. Therefore, a novel technique to compute the mean aerodynamic forces and power should be used rather than conventional methods that provide direct estimations. In this study, an ANN is employed to provide rapid predictions of the fitness function. The training data for the ANN is obtained from the UVLM (Nguyen, Kim, Han, \& Han, 2016).

\section{Results and Discussion}

Optimal solutions corresponding to various different values of ascending speed are derived and presented in this section.

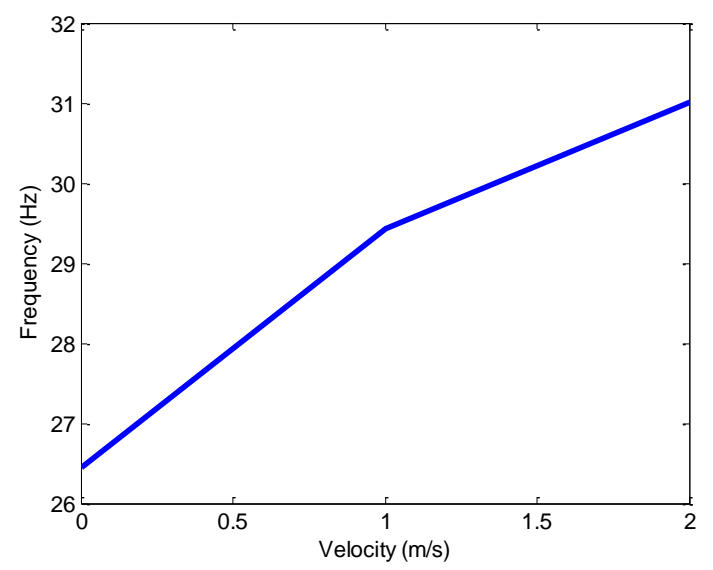

Figure 2: FWMAV and angle definitions

Figure 2 shows the variation of the flapping frequency against the ascending speed. It is seen that the frequency increases when the FWMAV moves upward faster. While hovering, the flapping frequency is $26.5 \mathrm{~Hz}$, which is close to $26.1 \mathrm{~Hz}$ of an actual hawkmoth recorded by Willmott and Ellington (Willmott \& Ellington, 1997). However, when the ascending speed is $2 \mathrm{~m} / \mathrm{s}$, this value goes up to about $31 \mathrm{~Hz}$. 


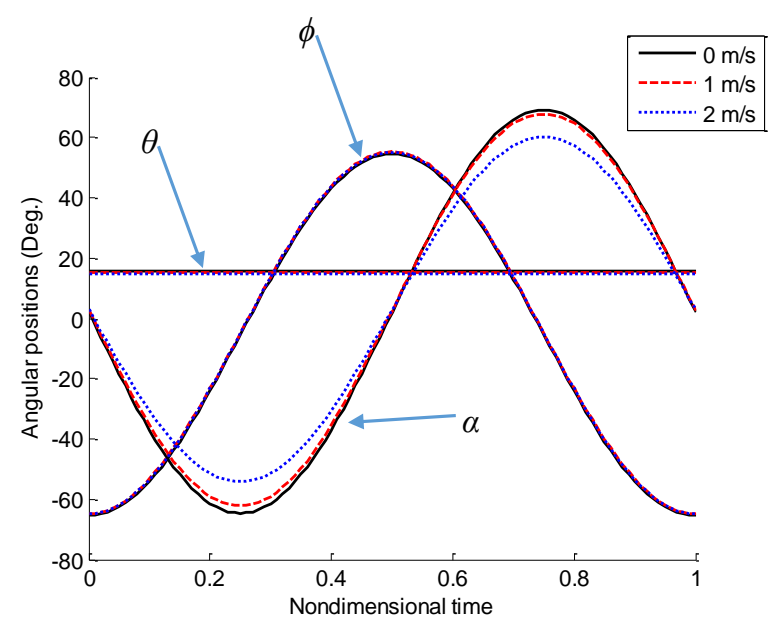

Figure 3: Wing kinematics against ascending velocity

With respect to the wing kinematic functions, it is observed that there are almost no changes in the variations of the sweep and elevation angles (Figure 3). The amplitude of the sweep angle is always at a maximum allowed value that is $60^{\circ}$ in this study in order to avoid possible overlapping between the left and right wings. It has been demonstrated that for flapping flight, minimum power is achieved when maximizing the amplitude of the sweep angle (Berman \& Wang, 2007). The only noticeable difference, which can be observed is in the rotation angle. For this angle, the amplitude decreases with the ascending speed.

Figure 4 shows how the ascending speed affects the optimal required power of the FWMAV. It is found that the power almost linearly increases with the ascending velocity. In hover, the FWMAV requires only approximately $70 \mathrm{~mW}$ to sustain flight, and this amount of power increases by more than 1.5 times to almost $115 \mathrm{~mW}$ at $2 \mathrm{~m} / \mathrm{s}$. From this result, it may be concluded that ascending flight consumes more energy than hover.

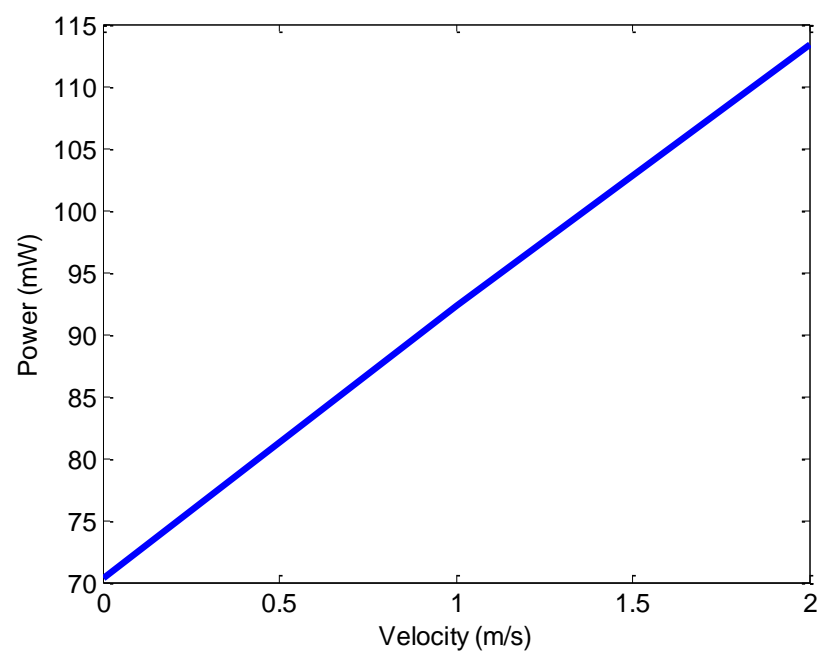

Figure 4: Required power against ascending speed 


\section{Conclusions}

This paper presents the optimal results of an insect-like FWMAV model while ascending. The optimization is carried out using the genetic algorithm, which is integrated with an artificial neural network to reduce the computational effort, as well as the running time. It is shown that when the ascending speed increases, the FWMAV has to move its wings at a larger frequency, and therefore, requires more power. The main difference in the wing kinematics of the FWMAV is found in the amplitude of the rotation angle. When the FWMAV ascending faster, the amplitude of the rotation angle is reduced.

\section{Acknowledgement}

This research is funded by Vietnam National Foundation for Science and Technology Development (NAFOSTED) under grant number 107.01-2018.05

\section{References}

Berman, G. J., \& Wang, J. Z. (2007). Energy-minimizing kinematics in hovering insect flight. Journal of Fluid Mechanics, 153-168.

Liu, H., Ravi, S., Kolomenskiy, D., \& Tanaka, H. (2016). Biomechanics and biomimetics in insectinspired flight systems. Philosophical Transactions of the Royal Society B: Biological Sciences, 20150390.

Mitchell, M. (1998). An introduction to genetic algorithms. MIT press.

Nguyen, A. T., Han, J. S., \& Han, J. H. (2017). Effect of body aerodynamics on the dynamic flight stability of the hawkmoth Manduca sexta. Bioinspiration \& biomimetics, 016007.

Nguyen, A. T., Kim, J.-K., Han, J.-S., \& Han, J.-H. (2016). Extended unsteady vortex-lattice method for insect flapping wings. Journal of Aircraft, 1709-1718.

Nguyen, A. T., Tran, N. D., Vu, T. T., Pham, T. D., Vu, Q. T., \& Han, J.-H. (2019). A NeuralNetwork-Based Approach to Study the Globally Energy-Optimal Hovering Wing Kinematics of an Insect Model. Journal of Bionic Engineering, 904-915.

Wei, S., Aono, H., Chimakurthi, S. K., Trizila, P., Kang, C.-K., Cesnik, C. E., \& Liu, H. (2010). Recent progress in flapping wing aerodynamics and aeroelasticity. sh Kumar Chimakurthi, Pat Trizila, C-K. Kang, Carlos ES Cesnik, and Hao Liu. "Recent progress in flapping wing aerodynamics and aeroelasticity." , 284-327.

Willmott, A. P., \& Ellington, C. P. (1997). The mechanics of flight in the hawkmoth Manduca sexta. I. Kinematics of hovering and forward flight. Journal of experimental Biology, 2705-2722. 